\title{
Verificação da abordagem do conteúdo de periodontia em provas de concursos públicos da Paraíba
}

\author{
Verification of the approach to periodontia content in public competition tests of Paraíba \\ Verificación del enfoque de contenido periodónico en las pruebas de concurso público de \\ Paraíba
}

Anaísa Teresinha Corcino Bidô ${ }^{1 *}$, Rodrigo Araújo Rodrigues ${ }^{1}$, Samara de Queiroz Ferreira Braga ${ }^{2}$, Rodrigo Alves Ribeiro ${ }^{1}$, João Nilton Lopes de Sousa ${ }^{1}$, Maristela Maia Lobo ${ }^{3}$, Ana Karina Almeida Rolim $^{4}$, Katryne Targino Rodrigues ${ }^{1}$, Elizandra Silva da Penha ${ }^{1}$, Rachel de Queiroz Ferreira Rodrigues ${ }^{1}$.

\section{RESUMO}

Objetivo: Verificar e analisar o conteúdo de periodontia abordado em concursos públicos da Paraíba através da análise e classificação de questões. Métodos: Foram extraídas questões que versavam sobre a área de periodontia de certames disponíveis realizados e encerrados na Paraíba para o cargo de cirurgião-dentista do Programa Saúde da Família entre os anos de 2001 e 2018. Estas, foram classificadas de acordo com o tópico genérico de que se tratavam e nível cognitivo que exigiam de acordo com a Taxonomia Revisada de BLOOM. Resultados: Foram recuperados 121 certames e extraídas 2.496 questões que correspondiam aos conhecimentos específicos ao cargo de cirurgião-dentista, destas, $156 ; 6 \%$, versavam sobre periodontia. Os resultados apontaram que o tópico de etiologia das doenças periodontais foi o de maior prevalência correspondendo a $23 \%$ das questões, seguido do tópico de diagnóstico, prognóstico e plano de tratamento com $21 \%$. Na classificação por dimensões cognitivas, lembrar foi a de maior ocorrência, correspondendo a $48 \%$. Conclusão: Questões que enfocavam a etiologia das doenças periodontais foram as mais abordadas em concursos públicos no contexto da periodontia e dimensões cognitivas mais complexas apresentaram baixa prevalência

Palavras-chave: Estratégia saúde da família, Seleção de pessoal, Periodontia.

\begin{abstract}
Objective: Objective: To verify and analyze the content of periodontics covered in public tenders in Paraíba through the analysis and classification of questions. Methods: Questions were extracted about the periodontics area of available contests held and closed in Paraíba for the position of dental surgeon of the Family Health Program between the years 2001 and 2018. These were classified according to the generic topic of what they were and the cognitive level they demanded according to the BLOOM Revised Taxonomy. Results: 121 events were recovered and 2,496 questions were extracted that corresponded to the specific knowledge of the position of dentist, of these, 156; $6 \%$ were about periodontics. The results showed that the topic of etiology of periodontal diseases was the most prevalent, corresponding to $23 \%$ of the questions, followed by the topic of diagnosis, prognosis and treatment plan with $21 \%$. In the classification by cognitive dimensions, remembering was the most frequent, corresponding to $48 \%$. Conclusion: Issues that focused on
\end{abstract}

\footnotetext{
1 Universidade Federal de Campina Grande (UFCG), Patos - PB. *E-mail: rachelperio@gmail.com

2 Centro Odontológico de Estudos e Pesquisas (COESP), João Pessoa - PB.

${ }^{3}$ Centro Universitário SENAC, São Paulo - SP.

${ }^{4}$ Universidade Estadual da Paraíba (UEPB), Campina Grande - PB.
} 
the etiology of periodontal diseases were the most addressed in public tenders in the context of periodontics and more complex cognitive dimensions showed low prevalence.

Keywords: Family health strategy, Personal selection, Periodontics.

\section{RESUMEN}

Objetivo: Verificar y analizar el contenido de la periodoncia cubierta en licitaciones públicas en Paraíba a través del análisis y clasificación de preguntas. Métodos: se extrajeron preguntas sobre el área de periodoncia de los concursos disponibles celebrados y cerrados en Paraíba para el puesto de cirujano dental del Programa de Salud Familiar entre los años 2001 y 2018. Estos se clasificaron según el tema genérico de cuáles eran y el nivel cognitivo que exigían según la taxonomía revisada BLOOM. Resultados: se recuperaron 121 eventos y se extrajeron 2.496 preguntas que correspondían al conocimiento específico del puesto de dentista, de estos, 156; $6 \%$ eran sobre periodoncia. Los resultados mostraron que el tema de etiología de las enfermedades periodontales fue el más frecuente, correspondiendo al $23 \%$ de las preguntas, seguido del tema de diagnóstico, pronóstico y plan de tratamiento con el $21 \%$. En la clasificación por dimensiones cognitivas, el recuerdo fue el más frecuente, correspondiente al $48 \%$. Conclusión: Los temas que se centraron en la etiología de las enfermedades periodontales fueron los más abordados en las licitaciones públicas en el contexto de la periodoncia y las dimensiones cognitivas más complejas mostraron una baja prevalencia.

Palabras clave: Estrategia de salud familiar, Selección de personal, Periodoncia.

\section{INTRODUÇÃO}

Após a promulgação da Constituição federal (CF) em 1988, o concurso público foi configurado elemento essencial na construção da administração pública no Brasil. O art. 37, inc. II, da CF destaca que a investidura em cargo ou emprego público depende de aprovação prévia em concurso público de provas ou de provas e títulos, de acordo com a natureza e a complexidade do cargo ou emprego, ressalvadas as nomeações para cargo em comissão declarado em lei de livre nomeação e exoneração (BRASIL, 1988). O concurso público foi escolhido por ser um processo seletivo impessoal onde é assegurada a igualdade de oportunidade dos profissionais que atuarão no serviço público (FERNANDES JUJ, 2018).

O concurso público é o meio orientado pelo Ministério da Saúde (MS) para seleção dos profissionais da estratégia de saúde da família (ESF), onde muitos candidatos são atraídos pela abundante oferta de vagas, pelos bons salários, e, principalmente, pela estabilidade. A estabilidade é a garantia de permanência no serviço, assegurada aos concursados, após três anos de exercício (SIQUEIRA FLB, 2018). No cenário atual o ingresso no serviço público constitui não apenas uma opção, mas sim, uma saída para enfrentar as dificuldades geradas pela instabilidade no mercado de trabalho (RIBEIRO CVS; MANCEBO D, 2013). Entretanto o que é observado atualmente é a presença do trabalho precarizado, marcado pela instabilidade advinda de contratações temporárias (OLIVEIRA RS, et al., 2015).

Concurso é um processo seletivo pautado pelos princípios constitucionais da legalidade, impessoalidade, moralidade, publicidade e eficiência (CARVALHO FILHO JS, 2014). Atualmente, a aplicabilidade de alguns desses princípios é questionável, seja em relação à igualdade de oportunidades, às suspeitas de fraudes levantadas constantemente, aos editais que não observam tópicos específicos e importantes da prática profissional ou aos métodos usados nas provas realizadas pelas bancas organizadoras, que tendem a repetir editais de acordo com os certames (MACÊDO DF et al, 2016).

Observa-se a necessidade de uma adequada seleção dos profissionais que atuam na ESF, pela importância que estes representam na melhoria da saúde bucal da população atendida, na resolutividade dos problemas encontrados e consequentemente, na eficácia da estratégia. Por conta disso, a literatura exibe o concurso público como a melhor forma de inserir o cirurgião dentista e outros profissionais de saúde na 
estratégia, em benefício da melhoria nos atendimentos, na satisfação dos usuários, no cumprimento da proposta da estratégia e na saúde da população (ARAÚJO MGGM, 2016; MOÇO LRS, et al., 2017; MACÊDO DF, 2016; VILELA EM e MAFRA LAS, 2015). Certames para contratação de pessoal para atuar na ESF, geralmente tem provas com questões de múltipla escolha, sendo um meio cujas habilidades necessárias para realizá-las em nada se assemelham às práticas de avaliação universitárias, e muito menos ainda às práticas profissionais inerentes ao cargo em disputa. Os certames devem articular preparação, realização prática, avaliação, organização e formação em torno de um processo que não seja mais desvinculado da noção de carreira (FONTAINHA FC, et al, 2014).

A periodontia é uma especialidade da odontologia responsável pelo periodonto, o conjunto dos tecidos de proteção e sustentação dos dentes, formado pela gengiva, osso alveolar, ligamento periodontal e o cemento radicular (NEWMAN N e NEWMAN MG, 2016). A doença periodontal é a segunda mais prevalente entre as doenças que acometem a cavidade bucal e, portanto, de grande importância para a dinâmica de atendimento na ESF (GONÇALVES ELM, 2010). Há alguns anos, um novo enfoque tem sido dado a doença periodontal, reconhecendo-a não apenas como problema nos tecidos de sustentação do dente, mas também como uma doença que pode apresentar uma relação direta com algumas doenças sistêmicas como as cardiopatias e patologias vasculares (ateroesclerose, acidente vascular cerebral ), além de, influenciar no nascimento de crianças com baixo peso ou prematuras (SANTANA FCM, et al, 2005; BRUNETTI MC, 2004).

$\mathrm{Na}$ área da Periodontia, na ESF são oferecidos tratamentos básicos como procedimentos de controle de placa supragengival (remoção de fatores retentivos - selamento de cavidades de cárie, extração de restos radiculares, acabamento e polimento de restaurações com excesso - e orientação de higiene bucal); raspagem subgengival de menor complexidade e tratamentos de urgência das lesões agudas do periodonto (GONÇALVES ELM, 2016). As diretrizes da política nacional de saúde bucal orientam que seja realizado o diagnóstico e tratamento das doenças periodontais (BRASIL, 2004).

Outrossim, pouco se conhece sobre a real abordagem do conteúdo de periodontia nas provas de concursos públicos, os assuntos mais frequentes e os domínios cognitivos exigidos dos cirurgiões-dentistas que pretendem ingressar na ESF e torna-se necessário analisá-los para se conhecer as principais exigências impostas ao provimento do cargo. Dito isto, objetiva-se com este estudo verificar e analisar o conteúdo de periodontia abordado em concursos públicos da Paraíba através da classificação das questões em tópicos genéricos de que se tratam e níveis cognitivos que exigem do participante do certame através da Taxonomia Revisada de BLOOM.

\section{MÉTODOS}

Trata-se de um estudo do tipo observacional, de abordagem quantitativa, analítica, documental e retrospectiva. Foi realizada uma busca, no período de agosto a setembro de 2018 , por provas de concursos públicos realizados e encerrados, de todas as cidades do estado da Paraíba para o cargo de cirurgião-dentista (CD) do Programa Saúde da Família (PSF) entre os anos de 2001 e 2018. Os exames foram recuperados, seguindo metodologia utilizada por Moço LRS, et al. (2017) e Penha ES, et al. (2020), por meio de pesquisa em um site de livre acesso utilizado por pessoas que realizam concurso público no Brasil, o PCI Concursos, encontrado através do portal eletrônico: www.pciconcursos.com.br/provas . Os descritores utilizados para a busca foram: cirurgião-dentista, dentista e odontólogo. Foram excluídos certames para cargos de especialistas na área de Odontologia, instrumentos idênticos aplicados entre os municípios e disponíveis de forma incompleta.

Aplicados os critérios, os testes foram catalogados, examinados um a um por dois pesquisadores e adicionados ao Microsoft Excel 2007. As questões foram analisadas extraindo das mesmas as que versavam sobre os assuntos da área de Periodontia, excluindo apenas as que apresentaram duplicidade. Em todas as etapas, o nível de concordância inter-avaliadores foi medido através do cálculo do coeficiente Kappa $(0,92)$, sendo 0,80 o mínimo valor aceitável para o estudo.

A classificação dos conhecimentos específicos por tópicos genéricos foi de acordo com a divisão do livro da versão mais recente de periodontia clínica do autor Newman N e Newman MG (2016), 12ª edição. Os 
tópicos abordados foram distribuídos da seguinte forma: 1 - anatomia do periodonto, 2 - classificação e epidemiologia das doenças periodontais; 3 - etiologia das doenças periodontais; 4 - patologia gengival e periodontal; 5 - tratamento não cirúrgico das patologias periodontais; 6 - diagnóstico, prognóstico e plano de tratamento; 7 - diagnóstico e tratamento das emergências periodontais; 8 - relação doença periodontal e saúde sistêmica; 9 - tratamento cirúrgico.

A taxonomia de objetivos educacionais de bloom classifica os objetivos pertencentes ao domínio cognitivo em seis níveis que, são apresentados numa sequência que vai do mais simples ao mais complexo: $\mathrm{i}$ conhecimento, ii - compreensão, iii - aplicação, iv - análise, v - síntese, vi - avaliação. As questões foram classificadas de acordo com a taxonomia de bloom revisada (tbr), onde as terminologias conhecimento, compreensão, aplicação, análise e avaliação foram substituídas pelas dimensões: $\mathrm{i}$ - lembrar, ii - entender, iii -aplicar, iv - analisar, v - avaliar e vi - criar, respectivamente (ferraz apcm e belhot rv, 2010).

As questões que continham subdivisões (itens $A, B$ e C), em relação aos critérios de classificação por níveis cognitivos foram analisadas como um todo, ou seja, todas as eventuais subdivisões foram analisadas como se fossem apenas uma pergunta, predominando a classificação que exigir um nível de cognição mais complexo segundo a Taxonomia. Em relação aos tópicos genéricos da periodontia as questões também foram analisadas como um todo, predominando a classificação do tópico genérico mais complexo.

Pelo não envolvimento de seres humanos e pelo material considerado de domínio público, não houve a necessidade de submissão ao comitê de ética. Mesmo assim, a pesquisa está em conformidade com as leis que regem o bom senso e a ética.

\section{RESULTADOS}

Foram coletadas 124 provas aplicadas entre 2006 e 2018. Ao aplicar os critérios de exclusão, foram removidas 3 provas idênticas, totalizando 121. Após análise destas, obteve-se 4.840 questões ao total, onde 2496 correspondiam aos conhecimentos específicos para o cargo de cirurgião-dentista e dessas 156; ou seja, $6 \%$, versavam sobre periodontia.

Ao examinar a prevalência de conhecimentos específicos por tópicos genéricos, constatou-se que o tópico referente à etiologia das doenças periodontais, que engloba a patogênese periodontal e os fatores predisponentes, foi o de maior ocorrência nos certames, correspondendo a $23 \%$ das questões, seguido do referente à diagnóstico, prognostico e plano de tratamento equivalente à $21 \%$ e anatomia do periodonto correspondendo a $14 \%$ das questões (Gráfico 1).

Gráfico 1 - Análise da classificação dos conhecimentos específicos por tópicos genéricos.

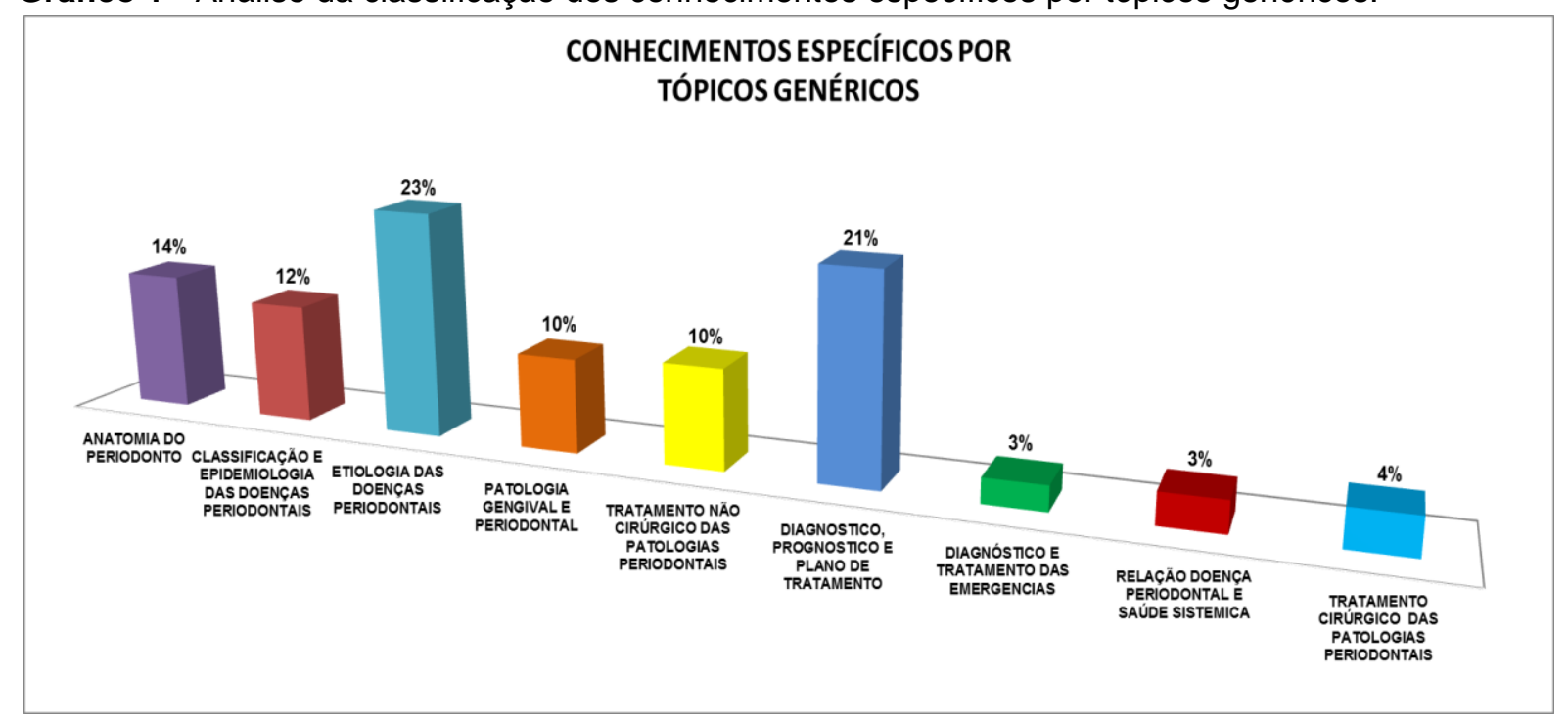

Fonte: Bidô ATC, et al., 2020. 
$\mathrm{Na}$ análise através da Taxonomia Revisada de Bloom (TRB), foi observado que $48 \%$ destas estavam inclusas na dimensão lembrar, onde o objetivo esperado dos alunos é basicamente verificar o que o mesmo já sabe, por reconhecimento ou memória, e $29 \%$ na dimensão aplicar que é relacionado a executar ou usar um procedimento em uma situação especifica ou nova (Gráfico 2).

Gráfico 2 - Análise da classificação por dimensões cognitivas da TBR.

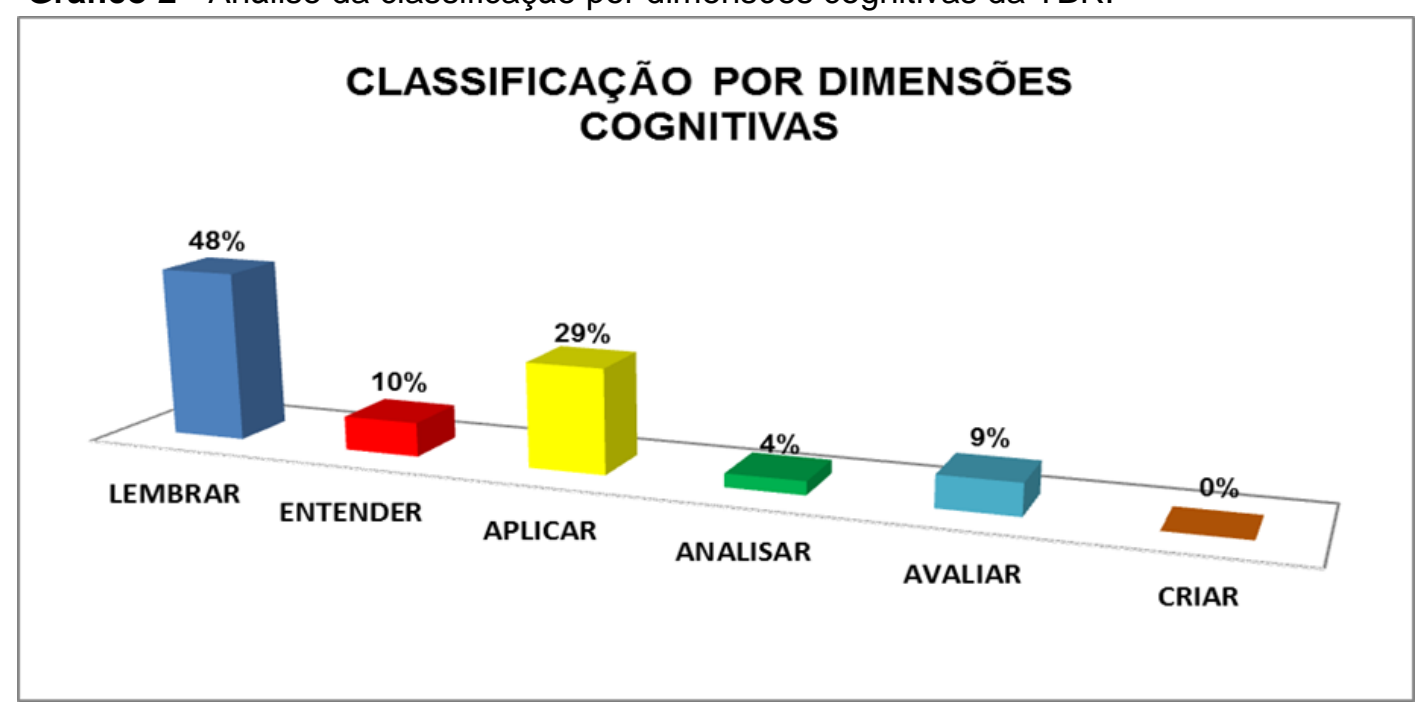

Fonte: Bidô ATC, et al., 2020.

\section{DISCUSSÃO}

Com o objetivo de ampliar o acesso aos serviços de saúde bucal, em 2001, ocorreu a implantação da equipe de saúde bucal (ESB) no PSF, através da portaria de março de 2001 (BRASIL, 2001). A partir disto, pode-se verificar a contratação de profissionais para compor o PSF e a inclusão da seleção destes em concursos públicos.

Neste estudo foram recuperadas 124 provas de concursos públicos realizados e encerrados no estado da Paraíba através do site PCl concursos. Sendo o meio de pesquisa escolhido, pois, trata-se de um dos maiores sites brasileiros especializado em certames públicos, sendo atualizado diariamente, contabilizando milhares de vagas em editais de todo o Brasil, além de recursos didáticos para aqueles que buscam a aprovação, sendo de acesso gratuito (KAIZER CD e SANTOLIN CB, 2018). As provas recuperadas foram realizadas entre os anos de 2006 e 2018, e não foram encontradas provas em ano anterior a 2006. Isso não quer dizer que não tenha ocorrido concurso público antes de 2006 no estado, apenas que essas provas podem não terem sido disponibilizadas no meio de pesquisa utilizado.

A atenção primária, por meio da ESF, é uma das prioridades do Sistema Único de Saúde (SUS) (BRASIL, 2014). Estudo realizado por Pinheiro VC, et al. (2011) analisaram o mercado de trabalho dos recém-egressos do curso de Odontologia da Universidade Federal do Ceará, da Faculdade de Farmácia, Odontologia e Enfermagem do ano de 2007, verificando a situação profissional, a jornada de trabalho, tipo de inserção no trabalho e a satisfação com relação ao mercado de trabalho. Observou-se, que a maioria, $27(46,5 \%)$, dos profissionais estão trabalhando no serviço público e quanto a forma de atuação profissional, apresenta-se com 24 profissionais $(41,4 \%)$ atuando como cirurgiões-dentistas no serviço público da ESF, mostrando a importância de avaliar as provas de concursos públicos para inserção do cirurgião-dentista no serviço público de saúde

Assim avaliar as questões dos certames torna-se imprescindível partindo da premissa de que as provas devem ser elaboradas e fundamentadas de acordo com o perfil profissional para a atuação no serviço público de saúde. Torna-se assim importante avaliar o perfil das questões da área da especialidade de periodontia para que os candidatos ao cargo conheçam a real abordagem dos conteúdos correspondente a essa 
especialidade, que são cobradas no concurso em disputa contribuindo para sua preparação e posterior aprovação.

$\mathrm{Na}$ ESF são oferecidos os procedimentos preventivos coletivos e individuais, dentística restauradora, cirurgia oral básica, endodontia básica e de periodontia. Na área da Periodontia, são oferecidos tratamentos básicos como procedimentos de controle de placa supragengival (remoção de fatores retentivos - selamento de cavidades de cárie, extração de restos radiculares, acabamento e polimento de restaurações com excesso - e orientação de higiene bucal); raspagem subgengival de menor complexidade e tratamentos de urgência das lesões agudas do periodonto (GOLÇALVES ELM, 2010).

Dentre as questões recuperadas, $6 \%$ relacionavam-se ao conteúdo de periodontia, evidenciando uma baixa prevalência deste assunto em certames onde o CD apto ao cargo realizará procedimentos dessa especialidade em seu cotidiano profissional. Esse achado concorda com o estudo realizado por Araújo MGGM (2019) que verificou o perfil de provas de concursos públicos de Odontologia no estado da Paraíba, entre os anos de 2007 a 2015, ao avaliar as questões técnicas relacionadas a área da odontologia, constatou que a periodontia possui uma prevalência de $9,6 \%$, ou seja, uma baixa prevalência.

Siqueira GRS (2014) ao avaliar a demanda de procedimentos odontológicos realizados na atenção básica do município de Rio Grande, Rio Grande do Sul, a partir de dados secundários da ficha do sistema de informação ambulatorial (SIA/SUS) do município, constatou que os procedimentos mais executados na ESF e na unidades básicas de saúde tradicionais, entre os anos de 2011 a 2013, foram a primeira consulta programática, verificada em $17,8 \%$ das fichas avaliadas, seguida dos procedimentos periodontais com $14,7 \%$, do total e os procedimentos restauradores e exodontias $11,6 \%$ e $10 \%$ respectivamente.

Pode-se inferir que a prevalência do conteúdo de periodontia nos concursos públicos não é correspondente com a frequência dos procedimentos mais executados pelos CDs na ESF. Uma vez que a periodontia está entre os procedimentos mais realizados na atenção básica, neste estudo. Entretanto, para que haja comprovação dessa associação são necessários estudos mais detalhados e que envolvam todo território nacional, visto que a maioria engloba apenas regiões específicas.

É importante reconhecer a importância da abordagem periodontal na ESF, visto que a doença periodontal é considerada um dos principais agravos em saúde bucal e classificada como a segunda maior patologia bucal que acomete a população mundial (GOLÇALVES ELM, 2010). Além disso, um novo enfoque tem sido dado à doença periodontal ao se reconhecer sua relação com algumas doenças sistêmicas como cardiopatias, patologias vasculares, diabetes, doenças respiratórias, nascimento de bebês prematuros e de baixo peso, entre outras.

A maior prevalência de conhecimentos específicos por tópicos genéricos foi referente ao tópico de etiologia das doenças periodontais, correspondendo a $23 \%$ das questões, seguida do tópico de diagnóstico, prognóstico e plano de tratamento, com $21 \%$. Este resultado é condizente com a prática clínica, na ESF, considerando a importância do diagnóstico e tratamento das doenças periodontais na atenção básica.

Ferreira ACR, et al. (2013) ao entrevistarem CDs do município de Vassouras, Rio de Janeiro, observou que $92 \%$ dos entrevistados atuantes no PSF relataram executar tratamento periodontal nos seus pacientes. Silva LS, et al. (2013) ao analisarem os dados do sistema de informações ambulatoriais do SUS no período de 2001 a 2010, mostrou resultado semelhante, onde o tratamento periodontal, juntamente com o restaurador foram os procedimentos mais executados na atenção básica entre os anos de 2001 a 2010 em todos municípios do estado do Pará. A análise desses estudos permite inferir que os principais tópicos de periodontia exigidos em provas de concursos na Paraíba são condizentes com os serviços periodontais mais executados na atenção básica primaria.

$\mathrm{Na}$ análise utilizando a TRB, as dimensões cognitivas lembrar e aplicar foram as mais frequentes, em detrimento das dimensões avaliar e criar, que praticamente não apareceram. Esse achado concorda com estudo realizado por Penha ES, et al. (2020) que analisou questões de Odontopediatria em concursos públicos do estado da Paraíba, classificando-as de acordo com a TRB, e obteve nos resultados como dimensões mais prevalentes as de lembrar (24\%) e aplicar (15\%), não encontrando representatividade das 
dimensões avaliar e criar. Tais resultados permitem inferir que em provas de concurso público na Paraíba para o cargo de $C D$, as questões requerem dos candidatos principalmente, conhecimento e compreensão.

Moimaz SAS, et al. (2017) analisaram e classificaram as questões de saúde coletiva do Exame Nacional de Desempenho de Estudantes (ENADE) do curso de odontologia nos anos 2004, 2007, 2010 e 2013 utilizando a TRB, e observaram que as dimensões analisar e avaliar, que exigem dimensões cognitivas mais complexas foram as mais prevalentes, em detrimento das dimensões lembrar, entender e aplicar, consideradas dimensões menos complexas. Esta discordância pode se justificar pelo fato de os exames possuírem objetivos diferentes. O ENADE apresenta objetivos meramente educacionais, enquanto o concurso objetiva medir o conhecimento do profissional apto ao cargo.

Fontainha FC et al. (2014) ao avaliarem 698 certames aplicados por 20 instituições públicas federais em todo Brasil, observando os componentes dos editais, as bancas organizadoras, o perfil dos candidatos. Observou que no que se refere à forma de seleção, a forma predominante, foi através da aplicação de questões de múltipla escolha, e não sendo observada a realização de provas práticas.

Pode-se concluir que esse modo de seleção avalia apenas a capacidade do candidato de encarar a forma avaliativa exclusiva do certame, e que em nada se assemelha a prática profissional, sendo necessário avançar em novas práticas na realização de concursos público.

Com isso infere-se as limitações desses processos seletivos, onde o certame, seleciona pessoas que não são nem os melhores profissionais experimentados nem os melhores egressos do sistema de ensino. São recrutados aqueles que têm mais sucesso na realização de provas. Deve-se ressaltar que essas análises não permitem a avaliação da qualidade dos concursos públicos realizados no estado da Paraíba, mas apenas a natureza das questões propostas nas provas.

\section{CONCLUSÃO}

Conclui-se que as questões mais frequentes na área da periodontia em concursos públicos da Paraíba, de acordo com o tópico genérico de que se tratam são referentes ao tópico de etiologia das doenças periodontais, seguido do tópico de diagnóstico, prognóstico e plano de tratamento, evidenciando a importância do profissional apto ao cargo saber diagnosticar e tratar as principais doenças periodontais, conforme recomendações das diretrizes da política nacional de saúde bucal em vigência no Brasil. As dimensões cognitivas lembrar e aplicar, que requerem memória e que o participante reconheça e reproduza ideias e consiga executar ou usar um procedimento em uma situação específica ou nova, respectivamente foram as de maior prevalência, em detrimento da dimensão criar, que não apresentou representatividade nesse estudo, visto ser, a dimensão de maior nível a ser exigida de um participante, que está relacionada ao desenvolvimento de ideias novas e originais, produtos e métodos por meio da percepção da interdisciplinaridade e interdependência de conceitos e de difícil colocação neste tipo de certame, que possui geralmente apenas questões de múltipla escolha.

\section{REFERÊNCIAS}

1. ARAÚJO MGGM, et al. Qual conteúdo é abordado em um concurso público em odontologia? Brazilian Journal of Development, 2019; 5(10): 19572-19585.

2. BRASIL. Constituição (1988). Constituição da República Federativa do Brasil. Brasília, DF: Senado Federal, 1988.

3. BRASIL. Ministério da saúde (MS). Guia Prático do Programa Saúde da Família. Ministério da Saúde, 2001.

4. BRASIL. Ministério da Saúde (MS) Diretrizes da política nacional de saúde bucal. Ministério da Saúde, 2004.

5. BRASIL. Ministério da saúde (MS). Portaria no 1.646, de 5 De agosto de 2014. Institui o componente GraduaCEO BRASIL SORRIDENTE, no âmbito da Política Nacional de Saúde Bucal, que irá compor a Rede de Atenção à Saúde (RAS), e dá outras providências. Diário oficial da união, 2014.

6. BRUNETTI MC. Periodontia Medica. Uma Abordagem Integrada. São Paulo: Editora Senac são Paulo, 2004.

7. CARVALHO FILHO, J. S. Manual de Direito Administrativo. 27 ed. São Paulo: Atlas, 2014.

8. FERNANDES JUJ. Proibição à realização de concursos e nova proposta para seleção de servidores. Rev JUS Navigandi [revista em internet] 2018 janeiro [acesso 3 de maio 2018]; 23(5321). Disponível em: <https://jus.com.br/artigos/63621>. 
9. FERRAZ APCM, BELHOT RV. Taxonomia de Bloom: revisão teórica e apresentação das adequações do instrumento para definição de objetivos instrucionais. Revista Gestão e Produção, 2010; 17(2):421- 431.

10. FERREIRA ACR, et al. Doença periodontal: um mal que pode ser evitado. Revista sobrape, 2013; 23(03):15-23.

11. FONTAINHA FC, et al. Processos seletivos para contratação de servidores públicos: Brasil, o país dos concursos? Rio de Janeiro: FGV Direito Rio, 2014.

12. GONÇALVES ELM. A importância da intervenção em doença periodontal pela equipe de saúde da família. Monografia (Curso de Especialização em Atenção Básica em Saúde da Família) - Universidade Federal de Minas Gerais, Uberlândia, 2010, 35p.

13. KAIZER CD, SANTOLIN CB. Conteúdos de provas de Educação Física em certames públicos dos Institutos Federais. Educação Física em Revista, 2018; 10(3):23-34.

14. MACÊDO DF, et al. Análise do concurso público como instrumento de seleção de pessoal no setor público: percepção de um grupo de servidores de instituições federais de ensino superior. Revista Sociais e Humanas, Santa Maria, 2016; 29(1): 92-110.

15. MOÇO LRS, et al. Características dos concursos para contratação de cirurgiões-dentistas na estratégia saúde da família. Revista ABENO, 2017; 17(2):22-31.

16. MOIMAZ SAS, et al. Enade em odontologia: análise e reflexões à luz da taxonomia de Bloom revisada. Revista ABENO. 2017; 17(3):30-40.

17. NEWMAN N, NEWMAN MG. Carranza Periodontia Clínica.12. ed. Elsevier Brasil, 2016.

18. OLIVEIRA RS, et al. Relações contratuais e perfil dos cirurgiões-dentistas em centros de especialidades odontológicas de baixo e alto desempenho no Brasil. Saúde e sociologia, 2015; 24(3):792-802.

19. PENHA ES, et al. Conteúdo em odontopediatria em exames da função pública no estado da paraíba: como esta especialidade está sendo abordada? Bioscience Journal, 2020; 36(1):304-309.

20. PCI CONCURSOS. Disponível em: < www.pciconcursos.com.br/provas > Acesso em: 20 de ago. de 2018.

21. PINHEIRO VC, et al. A inserção dos egressos do curso de odontologia no mercado de trabalho. Rev. Gaúcha de Odontologia, 2011; 59(02):277-283.

22. RIBEIRO CVS, MANCEBO D. O servidor público no mundo do trabalho do século XXI. Psicologia, Ciência e Profissão, São Paulo, 2013; 33(1): 192-207.

23. SANTANA FCM, et al. A doença periodontal como fator de risco em gestantes com bebês prematuros de baixo peso ao nascer. Pesquisa brasileira odontopediatria clínica integrada, 2005; 5(3): 247-252.

24. SILVA LS, et al. Indicadores de atenção básica e especializada em saúde bucal nos municípios do estado do Pará, Brasil: estudo ecológico, 2001-2010. Epidemiologia e Serviços de Saúde, 2013; 22(2): 325-334.

25. SIQUEIRA FLB. Reflexos da nomeação tardia em concurso público. Revista Científica ICGAP, 2018; 1(1).

26. SIQUEIRA GRS. Procedimentos Odontológicos: um estudo de demanda em unidades básicas de saúde no município do Rio Grande, RS. Dissertação (Mestrado em Saúde Pública) - Universidade Federal de Pelotas, Pelotas, 2014. $46 \mathrm{p}$.

27. VILELA EN, MAFRA LAS. Estratégia saúde da família: contratação temporária e precarização nas relações de trabalho. Caderno de Estudos Interdisciplinares, 2015. 